specific methods for measuring circulating peptides are being perfected, and hopefully new information will soon be available.

There are useful chapters on abnormalities of skin and of the central nervous system, that describe the clinical pictures seen in various tumours associated with these entities, but the chapter on "skeleton and ossification" could have been more detailed considering the great interest in this subject over the past decade.

Finally, there is an extremely useful reference list, including the more important publications.

This book is a well-presented, easily read monograph that should be useful to all clinicians who deal with cancer patients. Hopefully experimental biologists and biochemists will make use of it, since the chapters pose, for the scientist, many fascinating clinical questions that demand an answer.

R. C. СоомвеS

\section{Progress in Clinical and Biological}

Research: Vol. 25. Recent Advances in Clinical Oncology. Eds. T. A. HazRA and M. C. Beachley (1978). New York: Alan R. Liss. 180 pp. $\$ 16.00$.

This book is a collection of the papers presented at a conference in Williamsburg in February and March 1977.

The contributors are all well known in the field of oncology.

Four different subjects were covered. The first group of papers dealt with non-Hodgkin's lymphoma. The paper on the pathology summarizes the present classification of these tumours. The next paper, on the diagnosis and clinical manifestations, covers all the possible ways in which this group of tumours may be seen, and lists the procedures which need to be adopted for diagnosis and staging. The place of staging laparotomy is discussed in the next paper, and is summarized well in the final sentence :"Staging laparotomy has a highly selected but infrequent place in the management of the patient with nonHodgkin's lymphoma." Then there are 2 chapters on the treatment of this lymphoma, summarizing the 2 authors' experience with chemotherapy and radiotherapy. Finally in this section is a description of the condition in children, the way it differs from nonHodgkin's lymphoma in adults, and the improved survival which has been achieved with intensive chemotherapy.

The second group of papers deals with cranial tumours, mainly in children, and includes the role of radiotherapy in acute lymphoblastic leukaemia. The first of the 3 papers in this section reviews the use of the CT Scanner in diagnosing brain tumours in children. The second paper concerns the radiotherapy and chemotherapy of some brain tumours in children and adults. The final paper reviews the radiation treatment used as prophylaxis for CNS disease in acute lymphoblastic leukaemia, and rightly ends by stressing the need for further improvements in the management of this disease, despite the great improvements already achieved.

The third group of papers relates to genitourinary tumours, picking out some aspects of the management of this group of tumours. The value of radiation therapy in carcinoma of the prostate is assessed and future prospects discussed. The next paper reviews the progress that has been made in the treatment of testicular tumours with chemotherapy, but mention is also made of the use of this modality in other urogenital tumours. The third paper in this group describes the combined radiotherapy and surgical approach to the management of T3 tumours of the bladder.

The fourth group is entitled "Physics in Radiation Oncology" and the first paper usefully describes the use of the computer in radiation therapy. The second paper describes the problem of mycosis fungoides and its clinical features and management, leading appropriately to the last paper about the physical aspects of electron beam therapy in its application to the treatment of mycosis fungoides.

This is an interesting group of papers on a variety of subjects, but seems expensive for a collection of papers many of which have been published elsewhere.

\section{Pearson}

\section{Fundamentals of Cancer Chemotherapy.}

M. D. Crindland (1979). Lanes: MTP Press. pp. 115. £7.95.

This short and much abbreviated book on Cancer Chemotherapy is directed at practitioners unfamiliar with the management of malignant disease by drug treatment, and at medical students. The clipped precise style of the author may not appeal to every reader, and the condensed style of the book leads to 
some curious emphases, which I suspect not all oncologists would agree with. If this book is truly intended for the general practitioner who has to deal with patients treated in a specialist hospital, who may develop the complications of chemotherapy at home, it is somewhat surprising that whilst we are informed that busulphan uncommonly causes bronzing of the skin and a wasting syndrome, we are not informed that it may cause cumulative and life-threatening marrow depression in the treatment of chronic myeloid leukaemia.

It is difficult also to place this particular volume in the ever extending library of books by medical oncologists. The format of the book makes it a little indigestible and I feel that it could have been substantially improved with tables and line diagrams.

Most volumes on cancer chemotherapy are out of date by the time of publication because of the rapid changes and this volume is no exception, with some of the references not being updated beyond the early 1970s. The rather negative conclusion of the author also makes the volume difficult to recommend, when she states in her concluding chapter that chemotherapy has little to offer. Certainly, few cures are achieved with chemotherapy at the present time, but the testimony of many patients to the very important palliation that they receive, sometimes over many years, must contradict this particular author's view. This reviewer would personally have valued cautious and rational optimism in conclusion, and I expect that general practitioners looking after patients with malignant disease might find that approach more valuable too.

H. Bush

Biological Markers of Neoplasia. Ed. R. W. Ruddon (1979). Amsterdam: Elsevier, North Holland. 590 pp. \$55.00.

This book is a collection of papers presented at the first International Conference on Biological Markers of Neoplasia held in Virginia, May 1978. The meeting covered a range of disciplines, with sections dealing with tumourassociated antigens, virus-associated cellsurface antigens, surface glycoproteins of neoplastic cells, ectopic hormones, DNAbinding proteins, plasminogen activators and enzyme biomarkers. It is an interesting and commendable decision to omit the oncofoetal markers such as CEA and $\alpha$-foetoprotein, since these have been so adequately summarized in a spate of recent publications. Rather the book concentrates on markers which frequently receive less attention but may with further development be of equal or greater importance, particularly since the discriminatory capacity of all but a notable few of the more conventional markers has been questioned.

The bias of this volume is very much towards the basic and developmental aspects of markers of neoplasia in model systems, and the first 2 sections examine the isolation, characterization and properties of cell-surface antigens of chemically and virally induced tumours, and considerable experimental detail is presented. A valuable feature is the inclusion of the whole discussion, so that controversy and variations of opinion become clear. The section on glycoproteins of neoplastic cells indicates the direction that the tumour work might take by examination of the chemistry of the histocompatibility antigens.

The section on ectopic hormones and enzyme biomarkers describes recent work relating to the usefulness of these markers in the management of human disease. Again, considerable biochemical detail is presented and newer methodologies revealed. The sensitivity and selectivity of each hormone or enzyme in malignancy is often the subject of considerable discussion.

Further sections dealing with the DNAbinding proteins and plasminogen activators return largely to the experimental models, examining changes associated with chemical carcinogenesis in a variety of animal species, with some contributions concentrating on human disease.

The introductory contribution to each section by recognized authorities ensures that the information is available to those less familiar with the detail. This book should be a most useful addition to any library, since it serves the dual purpose of presenting the latest information in the field and expanding the data base by including material of many parallel disciplines to which readers may not be generally exposed. As such it can be recommended to all interested in the newer markers of neoplasia, particularly for the clarity and quality of presentation.

B. M. Vose 